\title{
FUGA DE CEREBROS EN EL SECTOR SALUD ¿UN FENÓMENO QUE CONDICIONA EL DESARROLLO EN AMÉRICA LATINA?
}

\author{
María María Ibañez Martín * \\ Fernando Delbianco**
}

enviado: octubre 2013 - aceptado: junio 2014

\begin{abstract}
Resumen
El presente artículo tiene por objetivo encontrar variables que expliquen la fuga de cerebros en el sector salud para los países de América Latina. En el trabajo se realizan estimaciones econométricas para 20 países de América Latina, con objetivo de encontrar una explicación parsimoniosa de la fuga de cerebros y cómo puede afectar la mortalidad infantil o la esperanza de vida. Las conclusiones a las que se arriban varían según el método econométrico utilizado: panel ó mínimos cuadrados en tres etapas.
\end{abstract}

Clasificación JEL: I15, J4, J1

Palabras Claves: Fuga de cerebros, migración, América Latina

\begin{abstract}
This article aims to find variables that explain the brain drain in the health sector in Latin America. At work, are made econometric estimates for 20 countries in Latin America with purpose of finding a parsimonious explanation of the brain drain and how it can affect infant mortality or life expectancy. The conclusion differs depending on the estimation method used, panel or in three stages least squares.

JEL Classification: I15, J4, J1

Key Words: Brain Drain, migration, Latin America

*** $\quad$ mariamariaibanez@gmail.com - maria.ibanez@uns.edu.ar; 12 de Octubre 1198, Bahía Blanca
fernando.delbianco@uns.edu.ar; 12 de Octubre 1198, Bahía Blanca
\end{abstract}




\section{INTRODUCCION}

La migración calificada es uno de los temas de mayor interés tanto para los países expulsores de recursos como para países receptores. La "desterritorialización" del mundo científico no constituye un fenómeno nuevo; por el contrario, su universalidad es una cualidad intrínseca a su existencia (Pellegrino y Vigorito, 2009). La migración calificada en América Latina comienza a mediados del siglo XVII, junto con lo conocido como "primera globalización" y se extiende a lo largo de la historia con períodos de mayor caudal de emigración.

La fuga de cerebros es considerada por algunos investigadores como un fenómeno fuertemente nocivo para los países expulsores, sosteniendo enérgicamente que coarta las posibilidades de desarrollo. Por otro lado hay autores que resaltan el aspecto positivo para el país de origen, prestando mayor atención a la posibilidad de que el fenómeno se revierta luego de que los profesionales adquieran mayor formación y logren nutrir al país en su regreso ó el efecto positivo que generan las remesas. Lo que finalmente se observa, al estudiar con detenimiento el fenómeno de fuga de cerebros, es que no hay un consenso respecto a los efectos que genera en los países expulsores de recursos como tampoco respecto las políticas que deberían implementarse en materia migratoria.

La presencia del fenómeno en el sector salud juega un rol fundamental. Los países en desarrollo presentan graves falencias en sus sistemas de salud y gran parte de ellas se deben a la falta de médicos y enfermeras. Estudios han relevado el éxodo de médicos que soportan dichos países pero la realidad es que los estudios empíricos realizados hasta el momento son escasos y distan de tener una importante validez externa (Docquier y Moullan, 2010). El objetivo del presente trabajo es identificar las causas de la fuga de cerebros en el sector salud para los países de América Latina. El análisis tiene por objeto realizar una estimación que permita explicar la presencia del fenómeno y medir la incidencia del fenómeno en el desarrollo económico de los países bajo análisis.

El trabajo se organiza según el siguiente esquema: en la primera sección se hace un análisis de la migración calificada en el caso específico de América Latina, mientras que en la segunda sección se evalúan los antecedentes para Argentina. En la tercera parte se analizan las distintas visiones sobre el impacto en el país de origen; en la cuarta se describen algunos determinantes de la fuga de cerebros. La quinta sección analiza el caso específico del sector de salud, donde se realizan estimaciones mediante metodología de panel y mínimos cuadrados en tres etapas. Por último, se expresan las conclusiones del trabajo. 


\section{LA MIGRACION CALIFICADA EN AMERICA LATINA}

La migración internacional, en sus momentos de mayor auge, fue acompañada por la agudización de los procesos de globalización. La primera etapa de auge migratorio tuvo correlación con la expansión europea en el mundo. Ésta etapa de primera globalización duró aproximadamente un siglo y se estima que los europeos que migraron completaron los 52 millones, siendo los principales destinos: América del Norte, América del Sur, África del Sur y Oceanía. La segunda globalización se expandió desde la segunda mitad del siglo XX hasta la actualidad. En éste período la migración posee características peculiares, dado que los países pobres son los países expulsores de población y los más desarrollados son los países receptores (Pellegrino y Vigorito, 2009).

Hubo entre ambos periodos un lapso de tres décadas en el cual la migración se detuvo y las políticas migratorias en los países receptores tomaron un carácter restrictivo. En la década de 1970 hubo en América Latina un punto de inflexión en materia migratoria. La crisis del modelo de "sustitución de importaciones" tuvo como consecuencia el aumento de la inquietud social y estallidos que concluyeron, en varios países, en una instalación de regímenes militares altamente represivos, acompañados de un incremento de la emigración de latinoamericanos (Pellegrino y Vigorito, 2009). Entre los emigrados se encontraban personas con altos niveles de capacitación que pertenecían a las elites nacionales de oposición a los regímenes militares. Aunque la migración de científicos y especialistas no es un hecho nuevo en la historia de la Humanidad, es a partir del fin de la Segunda Guerra Mundial y paralelamente al proceso de descolonización en África, Asia y el Caribe (en las décadas de 1950 y 1960) cuando el fenómeno de la migración de personal calificado adquiere relevancia en el ámbito académico y en las discusiones de los organismos internacionales. Para entonces, los países desarrollados y muchos no desarrollados tuvieron entre sus prioridades la reestructura y la creación de universidades y centros de investigación (Pellegrino y Vigorito, 2009).

De acuerdo con los datos reunidos por IMILA, el número total de técnicos, profesionales y afines que integran el stock de migrantes regionales en el continente se duplicó entre 1970 y 1980, involucrando 220 mil personas; en el decenio siguiente la magnitud absoluta del aumento fue algo menor, pues su total llegó a poco más de 300 mil efectivos en 1990.

En América Latina, la masificación de la migración internacional hacia Estados Unidos y más recientemente hacia España y otros en países desarrollados, tuvieron impactos diversos sobre la economía, la sociedad y la cultura (Villa, 2001). Si 
bien el nivel educativo de los emigrantes latinoamericanos a los Estados Unidos es inferior al de la población nativa de este país, Pellegrino (2000) advierte — con arreglo a un esquema de Sassen (1988) — que su perfil de calificación se polariza en dos extremos: (a) el conjunto de personas altamente calificadas insertas en ocupaciones gerenciales y de ciencia y tecnología; (b) el grupo más numeroso que se concentra en subsectores económicos de baja productividad, aún si ello les significa cumplir funciones para las que pudieran estar sobrecalificados. Dicha polarización permite advertir que si bien los menos calificados predominan en el stock de inmigrantes de la región en los Estados Unidos - condición que puede verse exagerada por el tipo de ocupación declarada-, el número de aquellos que poseen un grado relativamente alto de calificación es considerablemente elevado en relación con la disponibilidad de tales recursos humanos en los países de origen. Este aspecto es apoyado por el hecho de que los profesionales y técnicos constituyen más del 15\% de los migrantes procedentes de Argentina, Venezuela y Chile. Aquí, podría pensarse que la migración puede verse afectada por una cuestión de ingresos y poder adquisitivo, dado que los profesionales son aquellos que se encuentran mejor posicionados para enfrentar el costo de un traslado internacional. Esta última cuestión, será objeto de futuras investigaciones dada la dificultad de encontrar bases de datos que permitan hacer un análisis de causalidad en este sentido.

Cuadro 1. Latinoamericanos en Estados Unidos

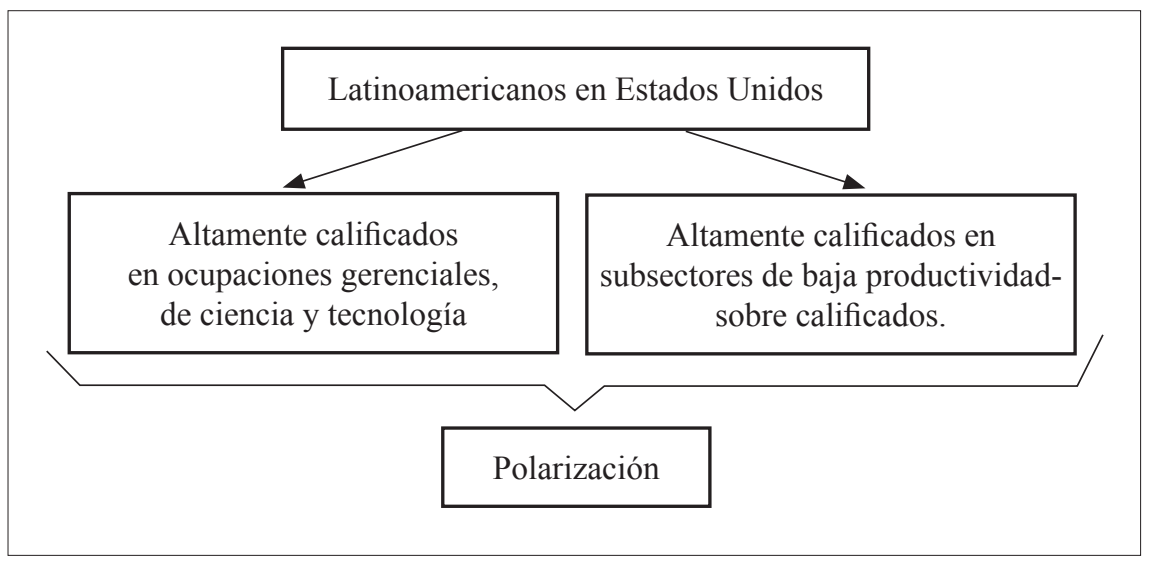

Fuente: Elaboración propia.

Docquier, Lohest y Marfouk (2005) resaltan que en la composición de la inmigración de alto nivel educativo predominan las corrientes de Europa y Asia del Sur y del Sudeste; sin embargo, las mayores tasas de "drenaje de cerebros" 
en relación a la fuerza de trabajo educada existente son las de América Central, el Caribe, los países de África Occidental y Oriental. Su análisis muestra que la migración calificada se ha incrementado de manera considerable durante la década de 1990: en los países de la OCDE el stock de inmigrantes calificados creció el 64\%. Es importante señalar que ese aumento fue sensiblemente mayor que el promedio para los originarios de países menos desarrollados (93\%), con un $113 \%$ para África y un 97\% para la región de América Latina y el Caribe. Es dable destacar que la comparación debe realizarse con cierto cuidado, los condicionantes políticos y económicos de cada región o país difieren ampliamente. Sin embargo no es objeto de la presente investigación ahondar en dichos acontecimientos, dado que el análisis que se presenta corresponde a una región con cierta homogeneidad en dichos aspectos.

\section{ARGENTINA: LA HISTORIA DE LA MIGRACION CALIFICADA.}

Argentina tiene una larga historia de migración calificada. Desde los años sesenta viene acompañando los ciclos de interrupción democrática, de deterioro económico y social del país. El caudal de emigración generó y genera una importante cantidad de argentinos en el exterior, llevando a instalar en la sociedad argentina la imagen del emigrante y validar la migración como una alternativa viable frente a las limitaciones internas para dar continuidad al progreso y ascenso social (Calvelo, 2008).

El surgimiento de la migración argentina puede ubicarse en la década del 60 ' y ha tenido continuidad desde entonces. Siguiendo a Calvelo (2008) podemos clasificar la historia migratoria de Argentina en las siguientes etapas:

I. La primera etapa se extiende en el periodo $1960-1975$ y es la que habitualmente se conoce como fuga de cerebros. Su ocurrencia tuvo lugar con el inicio del periodo de inestabilidad política, deterioro social y económico que tuvo lugar con las sucesivas interrupciones democráticas durante el gobierno de Frondizi e Illia.

En particular, la noche de los bastones largos abrió un ciclo de intervención y violación de la autonomía universitaria, marcando el final de una época de desarrollo científico y académico centrado en las universidades públicas.

Se inició entonces una importante migración de científicos e investigadores argentinos. Venezuela y Chile recibieron la mayor parte de los investigadores, docentes y científicos que se vieron obligados a renunciar a sus car- 
gos en institutos y universidades del país. Luego, Brasil, Francia y México fueron principales destinos de los profesionales argentinos que decidieron migrar por la insostenible situación del país.

II. El segundo período está asociado al exilio político y se extiende a lo largo del período 1976-1983, que coinciden con el inicio del golpe militar y el restablecimiento del gobierno democrático.

En el marco de las detenciones, intervención de los sindicatos, prohibición de huelgas, negociaciones colectivas y actividad política estudiantil de la última dictadura militar, se produjo una intensa salida internacional de militares, intelectuales, profesionales, científicos, estudiantes, artistas y gremialistas, con objetivo de escapar a la persecución política y de poner a salvo sus vidas.

En este periodo se diversificó la composición educativa y ocupacional de las corrientes hacia el exterior. Sin embargo, la calificación de los emigrantes argentinos continúo siendo elevada en el marco de la migración de latinoamericanos, que incluyó también figuras importantes de la investigación, las artes y la literatura.

Se destaca la presencia de grupos sociales intermedios, los grupos se diversificaron respecto a la primera etapa, incluyendo trabajadores manuales y trabajadores de oficios.

III. Con el deterioro económico generado en 1987 se inicia la tercera etapa, 1989-1992. Los saqueos y un nuevo suceso de fuga llevaron a miles de argentinos a emigrar hacia el exterior, como una manera de "huir" de la situación hiperinflacionaria del país.

En este caso, los mayores destinos fueron España e Italia como así también Estados Unidos, Canadá y Australia. La pérdida súbita de patrimonio y poder adquisitivo fueron las causas inmediatas de la nueva etapa de emigración argentina.

El perfil social de los emigrantes argentinos se diversificó aún más que en la etapa anterior. Distintos estratos de la clase media, trabajadores especializados y manuales, profesionales, pequeños comerciantes y jóvenes universitarios, participaron de esta migración voluntaria, principalmente motivada por la incertidumbre.

IV. La cuarta etapa comienza en 1998 y se extiende más allá de la crisis que sufrió Argentina en 2001. Aquí tuvo un papel primordial la acción de los medios de comunicación, que contribuyó a multiplicar el arrastre del flujo migratorio fortaleciendo la idea de "éxodo masivo". Los medios dan mayor 
visibilidad, pero debe probarse su relevancia (Novick, 2008). Se consolidaron nuevos destinos como España, Israel, Italia y Canadá, los cuales implicaban una mayor diversificación social y laboral de los emigrantes.

Notoriamente, Argentina tiene un proceso migratorio con olas de mayor expansión que concuerdan con la inestabilidad económica y social generada por las permanentes crisis de la economía.

\section{VISIONES SOBRE EL IMPACTO EN EL PAIS DE ORIGEN}

Si se observa la literatura respecto a la migración internacional de América Latina de los últimos años, se constata que las remesas que los migrantes envían a sus familiares o comunidades han sido el fenómeno más analizado. Su impacto en algunas economías de origen fue incluso considerado como una alternativa para estimular el desarrollo económico y como un factor positivo para reducir la pobreza. Algunos autores consideran a las remesas como un componente tangible del transnacionalismo. En este sentido, Villa y Martinez (2001) reconocen la importancia que tienen las remesas dentro de las migraciones verificadas en América Latina, mencionando el importante caudal de recursos financiero que significan las mismas. Por su parte Lim (1998) destaca el efecto que las remesas tienen sobre el rol que las mujeres desarrollan en los hogares, la existencia de éste flujo de recursos impulsa a la mujer a tomar decisiones sobre la administración familiar. La migración constituye una fuente de divisas para los países, comunidades y familias de origen. Samuel (2000) sostiene que las remesas pueden concebirse como parte de un arreglo contractual diferido en el tiempo, que confiere beneficios al migrante y al hogar en el país de origen. Tal arreglo involucra la inversión que la familia efectúa en uno de sus miembros - cuando afronta los costos de su educación- con la expectativa de que su migración permitirá obtener una compensación mediante las remesas, como un riesgo, que disminuirá en la medida en que ese miembro migre.

Por otra parte, la mayoría de los análisis coinciden en que la migración calificada apareja consecuencias negativas para los países de origen. Desde su postura, es evidente que la migración implica una pérdida de las inversiones que hacen los Estados en sus sistemas educativos para construir masas críticas de científicos, tecnólogos, profesionales y especialistas (Pellegrino, 2000).

Sin embargo, también existe una literatura reciente sobre migración calificada -en particular, altamente calificada- que pone el acento en la circulación de 
las personas y sus beneficios. Las expresiones brain drain (drenaje de cerebros) o éxodo de talentos, con que se designaba el fenómeno en los años sesenta, han tendido a ser reemplazadas por Brain Exchange o Brain Circulation, poniendo el acento en la movilidad que tiene lugar tanto desde como hacia los países desarrollados, así como la que tiene lugar entre los países en desarrollo. Se enfatizan las posibilidades de intercambio y de transferencias de conocimientos y habilidades que conlleva esta modalidad (Pellegrino y Vigorito, 2009). La idea de considerar la movilidad y la circulación como mecanismos para recuperar positivamente a los migrantes calificados para sus países de origen, se convirtió entonces en un leit motiv en algunas propuestas de políticas. Sin embargo, si bien es cierto que la movilidad y el intercambio son factores fundamentales para el desarrollo y la acumulación de conocimiento, las pérdidas definitivas siguen constituyendo un déficit importante que requiere primordial atención.

Podemos resumir los argumentos optimistas y pesimistas respecto al fenómeno analizado en la siguiente tabla, siguiendo a Pellegrino y Vigorito (2009).

Tabla 1. Argumentos optimistas de la Fuga de Cerebros

\section{Argumentos Optimistas}

La importancia que tienen las remesas sobre las economías nacionales y regionales.

Los efectos de las remesas sobre la reducción de la pobreza y del incremento en el bienestar de los hogares

Las remesas tienen como consecuencia el incremento en las inversiones domesticas que tienen efectos multiplicadores, directos e indirectos, de las transferencias monetarias realizadas por los emigrantes.

La emigración permite alivianar las tensiones producidas por el crecimiento demográfico y sus consecuencias sobre el empleo.

Las capacidades y habilidades adquiridas por los emigrantes y su transferencia al país de origen en caso de retorno, ó a través de los vínculos y las redes.

Fuente: Elaboración propia. 
Tabla 2. Argumentos pesimistas de la Fuga de Cerebros

\section{Argumentos de quienes tienen una visión negativa}

No hay evidencia alguna de que, por si solas, las remesas "desarrollen económicamente a un país que exporta fuerza de trabajo".

Las remesas de los emigrantes intensifican el consumo privado pero no estimulan las actividades productivas.

La inversión, de los emigrantes, en actividades productivas en sus países de origen ha tenido escaso impacto en el crecimiento económico de los países.

La emigración tiene consecuencias demográficas: despoblamiento y envejecimiento.

Los migrantes retornantes no encuentran, en la mayoría de los casos, ámbitos estimulantes para volcar los conocimientos adquiridos.

La migración "descapitaliza" la fuerza de trabajo del país de origen, que pierde personal calificado esencial para el desarrollo económico.

La emigración no reduce el desempleo porque no se van los desempleados sino aquellos que tienen posibilidades de insertarse en el mercado laboral del país receptor.

Fuente: Elaboración propia.

En referencia a los efectos generados por la fuga de cerebros, los mismos son vistos como positivos o negativos dependiendo de dos características principales: las acciones de gobierno en los países de origen y el carácter de la emigración. En lo que respecta al primer determinante, la creación oficial de centros de alto aprendizaje, el apoyo a proyectos de investigación y los incentivos financieros para el establecimiento de industrias privadas de alta tecnología pueden proporcionar la infraestructura necesaria para recibir y absorber las contribuciones de los profesionales emigrados. De no desarrollarse lo anteriormente mencionado, las buenas intenciones de los migrantes no contribuirían al desarrollo científico y tecnológico del país sino que solamente financiarían proyectos de "caridad". 
Respecto al carácter de la migración, cuando el movimiento es cíclico con viajes temporales en el extranjero pero con retornos a puestos permanentes en el país de origen, se eleva la potencialidad de transmisión de conocimientos y la transferencia de tecnología por efectos del fenómeno.

Portes (2007) señala que la migración permanente de profesionales no necesariamente tiene consecuencias negativas como sí lo tiene la de trabajadores manuales. En primer lugar, el fenómeno no genera una huída masiva de las áreas rurales debido a que en general proviene de las ciudades. En segundo término, aún cuando los profesionales en el extranjero puedan ser residentes permanentes e incluso convertirse en ciudadanos del país receptor, tienen la posibilidad de hacer que el proceso sea cíclico al utilizar sus recursos económicos y sus conocimientos para realizar transferencias regulares a su país de origen, inversiones cuantiosas o actividades programáticas en él.

A su vez, el autor destaca la importancia de que exista algo a que regresar. Si los emigrantes perciben que en los países de origen no tienen las mismas oportunidades que en el extranjero y que no existe una estructura tecnológica industrial ni un marco institucional claro, no hay incentivos para regresar. De este modo, la fuga de cerebros se convierte en un fenómeno de carácter negativo no habiendo transferencia de conocimientos ni de tecnología y las remesas generadas son mal utilizadas.

Stark (1998), Mountford (1997) y Beine, et al (2001) en sus respectivos estudios llegan a la conclusión de que la emigración calificada estimula el mejoramiento en los indicadores de educación de los países en desarrollo y que beneficia a los países de origen.

\section{VISIONES SOBRE LOS DETERMINANTES DE LA EMIGRACION CALIFICADA}

Los determinantes de los flujos de "fuga de cerebros" pueden enmarcarse dentro del análisis costo-beneficio y, por otro lado, dentro de la "nueva economía de la migración". En el primer caso, se encuentra respaldo debido a que la emigración calificada suele originarse en países pobres donde las remuneraciones esperadas son apenas una fracción de lo que pueden recibir en países desarrollados. Sin embargo, la teoría se ve contradicha por el hecho de que son las naciones con ingresos medios y no las pobres las que constituyen la fuente primordial de emigración de profesionales y, a su vez, dentro de estos países hay grandes varia- 
ciones en las probabilidades y motivaciones de la migración. Independientemente de las condiciones del país de origen, la mayoría de los profesionistas no toma la decisión de abandonar su tierra (Portes, 2007).

La "nueva economía de la migración" pone énfasis en la privación relativa que sufren los profesionales aspirantes a emigrar, teniendo dos grupos de referencia: los profesionales que en el país de origen están bien ubicados y los colegas en el extranjero con entrenamientos similares (Massey et. al, 2000). En el primer caso, la imposibilidad de alcanzar igual posición que los colegas dentro del mismo país es un poderoso motivador para emigrar. En otras palabras, la incapacidad para acceder a las remuneraciones que hacen posible un estilo de vida digno en sus propios países se convierte en un determinante clave de la fuga de cerebros. En el segundo grupo, el factor decisivo para la migración es la imposibilidad de conseguir condiciones y oportunidades laborales en el país de origen similares a las presentes en los países de destino (Portes, 2007).

Cuando se hace referencia a los determinantes de la migración calificada en América Latina es difícil identificar un patrón. La realidad es que la comparación permanente de las condiciones de vida de colegas, dado el progreso de los medios de comunicación, es inmediata. Por otro lado, los países de la región se caracterizan por la existencia de crisis periódicas que tiene un gran impacto en el poder adquisitivo, en la estabilidad económica, en la estabilidad social y en la libertad política de los individuos, la existencia de dichas crisis hace que las olas de migración sean cuantiosas y que en la mayoría de los casos estén formadas por individuos con alta calificación educativa y de un nivel social intermedio.

La dificultad de poder medir empíricamente algunos de los determinantes de la fuga de cerebros hace que las estimaciones empíricas tengan un bajo poder explicativo. En este contexto, el presente trabajo tiene el desafío de captar los determinantes de la fuga de cerebros.

\section{FUGA DE CEREBROS EN EL SECTOR SALUD}

En lo que se refiere específicamente a la emigración de los trabajadores del sector salud puede decirse que los estudios realizados son escasos y la revisión bibliográfica al respecto es compleja. En nuestro trabajo se tomó como referencia el estudio realizado por Bhargav, Docquier y Moullan en el año 2010. Los autores realizan una estimación de los efectos del fenómeno "medical brain drain" en el desarrollo humano para los países en desarrollo. 
Dentro de las conclusiones de su trabajo pueden destacarse que la oferta de médicos en los países en desarrollo mejoran los indicadores de desarrollo humano una vez que las tasas de alfabetización, en dichos países, superan el $60 \%$. A pesar de que la potencial migración incentiva la formación médica, la fuga de cerebros en el sector redujo los niveles de dotación en el país de origen no encontrándose evidencia de existencia del fenómeno conocido como "brain gain".

Por otro lado, concluyen que la reducción en la fuga de cerebros del sector médico disminuye la tasa de mortalidad infantil y aumenta la tasa de vacunación en los países en desarrollo. Sin embargo, tal efecto es demasiado pequeño como para considerarlo un factor decisivo para lograr las metas de desarrollo propuestas en el año 2008 por las Naciones Unidas.

Siguiendo la línea de investigación de los autores, lo propuesto en nuestro trabajo cambia el sentido, es decir, el objetivo principal es buscar la explicación del fenómeno medical brain drain y luego ver si tiene efectos sobre el desarrollo económico.

\section{V.1. Estimación del fenómeno medical brain drain para América Latina}

$$
\text { a- Datos: }
$$

Los datos utilizados para el trabajo corresponden a 20 países de América Latina. La lista de países contemplados es la enumerada en la tabla 2. Los datos provienen de dos fuentes. En primer lugar, se utiliza la base de datos utilizada por Bhargava, Docquier y Moullan (2010), disponible en el Banco Mundial. De dicha base, el foco se encuentra en la variable Medical Brain Drain (MBD), confeccionada por dichos autores. La variable se define como la fuga de cerebros en medicina en el momento i para el país t, siendo el ratio entre los médicos migrantes y los recibidos para cada país en cada momento del tiempo.

En segundo lugar, las restantes variables seleccionadas para el trabajo y las estimaciones realizadas, corresponden a datos disponibles en el sitio del Banco Mundial. El periodo abarcado por el análisis es, acorde a la base de datos usada de Bhargava, Docquier y Moullan (2010), desde 1991 a 2004.

\section{b- Metodología y Estimaciones}

Para las estimaciones, se tomaron dos caminos distintos. Por un lado, teniendo en consideración que son 20 países se procedió a estimar utilizando la 
metodología de panel de datos (usando especificaciones tanto de efectos aleatorios como fijos). Se especificaron errores convencionales, robustos y también calculados con bootstrap (para las tres estimaciones realizadas en el trabajo). Por otro lado, y para tener una alternativa de estimación a la de panel, se estima un sistema de ecuaciones mediante mínimos cuadrados en tres etapas (MC3E). En este caso el modelo seleccionado toma como base las regresiones realizadas por Beine, Docquier y Rapoport (2001).

Tabla 2. Países considerados en el análisis

\begin{tabular}{cc}
\hline Argentina & Guatemala \\
Bolivia & Honduras \\
Brasil & Jamaica \\
Chile & México \\
Colombia & Nicaragua \\
Costa Rica & Panamá \\
Dominica & Paraguay \\
Ecuador & Perú \\
Venezuela & Trinidad y Tobago \\
El Salvador & Uruguay \\
\hline
\end{tabular}

Fuente: Elaboración propia.

La estimación de panel tomará en consideración el tamaño de la base y las heterogeneidades no observadas de cada país (utilizando los errores de random effects o agregando fixed effects), mientras que por el otro lado, MC3E intentará capturar posibles simultaneidades y endogeneidades de las variables estudiadas. De esta manera, estimando vía dos metodologías visiblemente distintas, se intentará dar mayor robustez a los resultados hallados.

Entonces, bajo el contexto de panel de datos, el modelo seleccionado para intentar explicar MBD consiste en las siguientes variables explicativas:
I. Gasto Publico en Salud $\left(\% \mathrm{PBI}^{1}\right)$
II. Remesas recibidas ${ }^{2}(\% \mathrm{PBI})$
III. Crecimiento del PBI (\%)

\footnotetext{
1 Todas las variables extraídas del Banco Mundial se expresan en USD constantes.

2 Remesas de trabajadores y compensación de empleados recibidas (\% del PIB)
} 
IV. Número de patentes solicitadas por residentes

V. Número de artículos publicados en revistas científicas

VI. Gasto público en el sector educativo terciario (\%PBI).

Las variables correspondientes a patentes y revistas científicas fueron seleccionadas para este trabajo como proxy de la calidad académica y tecnológica del país, la cual puede estar condicionando la fuga de cerebros al estar definiendo en cierta medida el ambiente laboral de los profesionales de dicho país. El resto de las variables (crecimiento, gasto público en salud y remesas) son variables que se pueden observar también en el trabajo de Bhargava, Docquier y Moullan (2010), que también utilizan panel data para sus estimaciones.

Además, como se mencionó previamente, para tener una alternativa de estimación a la de panel se estima un sistema de ecuaciones mediante mínimos cuadrados en tres etapas (MC3E). En este caso el modelo seleccionado toma como base las regresiones realizadas por Beine, Docquier y Rapoport (2001). Dichos autores utilizan la migración como determinante de la formación de capital humano y el crecimiento económico. Para ello, estiman tres ecuaciones, cuyas variables dependientes son migración, capital humano y crecimiento. Las ecuaciones son estimadas de la siguiente manera:

Migración $=f($ Diferencial PBI-OCDE; Población $;$ Gasto Publico $)$ Educación $=f$ (Migración; Migración* Dummy ingreso bajo; Gasto Publico)

Crecimiento $=f$ (Migración $;$ Educación $;$ Remesas $)$

Donde Diferencial PBI-OCDE hace referencia a la diferencia de salario entre el país en cuestión y el promedio de países de la OCDE, y la Dummy de ingreso bajo es igual a 1 cuando el ingreso per cápita está entre el 15\% más pobre del mundo.

En relación a este sistema, se agregó una ecuación con MBD como dependiente y se eligió la siguiente especificación:

Migración $=f($ Diferencial PBI-OCDE; Población; Gasto Publico en Salud y Educación Terciaria)

Educación $=f$ (Migración; Gasto Público en Educación Terciaria)

Crecimiento $=f$ (Migración $;$ Educación $;$ Remesas; Crecimiento Poblacional)

$M B D=f($ Crecimiento; Educación; Migración; Gasto Salud $)$ 
Donde el Gasto Público fue focalizado en dos sectores: Educación Terciaria y Salud, pensando en su correlación con MBD.

\section{c- Estimaciones:}

\section{V.c.1. Panel:}

Entonces, una vez planteado el modelo a estimar y sus dos especificaciones definidas por la técnica econométrica a utilizar, se pasa ahora a reportar los resultados obtenidos.

Los resultados de la estimación realizada para MBD por estimaciones de panel se pueden observar en la tabla 3. En la misma se realizó además una especificación más simple omitiendo la variable de crecimiento y la de revistas publicadas (especificaciones 3 y 4). La única variable que resulta más robusta en las estimaciones realizadas es la correspondiente a las remesas. Además, el gasto en salud toma significatividad en las estimaciones realizadas con el marco de efectos fijos. El efecto que se obtiene es positivo, contario a lo que en principio uno esperaría, sin embargo podría sostenerse que la magnitud del efecto no compensa la desigualdad con las condiciones laborales con respecto a los países de destino.

A su vez, se estimaron dos modelos donde las variables dependientes son el logaritmo de la esperanza de vida y el logaritmo de la mortalidad infantil. Éstos intentan explicar por el gasto público en salud, el crecimiento y la variable MBD. De esta manera se intenta captar si MBD logra tener un impacto significativo en variables que indican el grado de desarrollo en materia de salud de un país (tabla 4). En estas estimaciones, más allá de la forma funcional elegida, MBD no resulta significativa para ninguna de las dos dependientes elegidas.

Más allá de la especificación seleccionada, la cual puede estar omitiendo variables (como el rol educativo en la mortalidad infantil, cuestiones políticas de los países de origen, de género, entre otros) y pecando de parsimoniosa, la fuga de cerebros de la rama de medicina no logra ser explicada satisfactoriamente por las variables escogidas por un lado, ni logra explicar las variables de salud elegidas por el otro. Sólo se consiguen significatividades del 5 o $10 \%$ en algunas especificaciones de variables explicativas de MBD, pero se pierden cuando se pasan a estimar los paneles con errores robustos o bootstrap.

En función de las estimaciones, se puede pensar que las variables seleccionadas no explican (por lo menos para los 20 países de América Latina selec- 
cionados) el MBD. Por otro lado, puede estar sucediendo que la fuga de cerebros también puede explicarse por las condiciones de los países receptores de la fuga. Es decir, quizás no sean las condiciones en sí de tal país las que alimenten la fuga de cerebros, sino la situación comparativa con los países que mayoritariamente reciben el flujo de profesionales.

Por otro lado, en esta sección, debido a las conclusiones encontradas podríamos pensar en la importancia que tomarían las variables omitidas. América Latina es una región con una gran diversidad de condiciones políticas, económicas y, específicamente, los países se diferencian en sus sistemas de salud. Sin embargo, debido a nuestro objetivo, decidimos seguir las especificaciones realizadas por Bhargava, Docquier y Moullan (2010). Serán futuras líneas de investigación, las estimaciones que incorporen los aspectos mencionados y análisis específicos para cada país.

Concluyendo, podemos sostener que el fenómeno MBD no es explicado por las variables seleccionadas en nuestro modelo de datos de panel, cuando nos ponemos estrictos en la confiabilidad. Por otro lado, la fuga de cerebros del sector salud no parece explicar de manera significativa las tasas de mortalidad infantil y de esperanza de vida, siendo su efecto prácticamente nulo. Finalmente, debido al resultado que obtuvimos podemos sospechar que el MDB puede explicarse mayoritariamente por las condiciones de los países de destino respecto a las que encuentran los profesionales de la salud en sus países natales, que será comprobado en futuras líneas de investigación.

Tabla 3. Efectos aleatorios (RE) y fijos (FE) sobre MBD.

\begin{tabular}{ccccc}
\hline \multirow{2}{*}{ MBD } & $(1)$ & $(2)$ & $(3)$ & $(4)$ \\
& $\mathrm{RE}$ & $\mathrm{FE}$ & $\mathrm{RE}$ & $\mathrm{FE}$ \\
\hline Crecimiento & 0.00025 & 0.00027 & & \\
& $(0.00029)$ & $(0.00019)$ & & \\
& & {$[0.00018]$} & & \\
Gasto en & $\{0.00057\}$ & & 0.00362 \\
Salud & 0.004383 & 0.004856 & 0.003215 & $(0.00180)^{*}$ \\
& $(0.00292)$ & $(0.00202)^{* *}$ & $(0.00332)$ & {$[0.00203]$} \\
& & {$[0.00226]^{*}$} & & $\{0.00497\}$
\end{tabular}




\begin{tabular}{|c|c|c|c|c|}
\hline \multirow[t]{4}{*}{ Remesas } & 0.0080 & 0.00342 & 0.0126 & 0.00349 \\
\hline & $(0.0025)^{* * *}$ & $(0.00191)^{*}$ & $(0.0028)$ & $(0.0019)^{*}$ \\
\hline & & [0.00234] & & {$[0.0025]$} \\
\hline & & $\{0.00380\}$ & & $\{0.0026\}$ \\
\hline \multirow[t]{4}{*}{ Patentes } & 0.000004 & -0.000003 & 0.0000 & -0.0000 \\
\hline & $(0.000011)$ & $(0.00000)$ & $(0.0000)$ & $(0.0000)$ \\
\hline & & {$[0.00000]$} & & {$[0.0000]$} \\
\hline & & $\{0.00000\}$ & & $\{0.000\}$ \\
\hline \multirow[t]{4}{*}{ Revistas } & 0.000000 & 0.00000 & & \\
\hline & $(0.000004)$ & $(0.00000)$ & & \\
\hline & & {$[0.00000]$} & & \\
\hline & & $\{0.00000\}$ & & \\
\hline \multirow{4}{*}{$\begin{array}{l}\text { Gasto en } \\
\text { educación } \\
\text { terciaria }\end{array}$} & 0.000428 & 0.00013 & 0.0006 & 0.0001 \\
\hline & $(0.000223)^{*}$ & $(0.00016)$ & $(0.0002)$ & $(0.000)$ \\
\hline & & [0.00013] & & {$[0.000]$} \\
\hline & & $\{0.00025\}$ & & $\{0.000\}$ \\
\hline \multirow[t]{4}{*}{ Constante } & 0.046033 & 0.05001 & 0.0290 & 0.0549 \\
\hline & $(0.032051)$ & $(0.0130)^{* * *}$ & $(0.0288)$ & $(0.0121)^{* * *}$ \\
\hline & & {$[0.0142]^{* * *}$} & & {$[0.0141]^{* *}$} \\
\hline & & $\{0.02151\}^{* *}$ & & $\{0.0275\}^{* *}$ \\
\hline
\end{tabular}

$\mathrm{N}=260$

$*, * *$ y $* * *$ : significativos al 10,5 y $1 \%$ respectivamente.

Desvíos estándar entre paréntesis, robustos entre corchetes y calculados con bootstrap entre llaves. 
Tabla 4. MBD como explicada.

\begin{tabular}{ccc}
\hline dependiente & Log $($ esperanza de vida $)$ & $\log ($ mortalidad infantil $)$ \\
\hline Crecimiento & $-1.69 \mathrm{E}-10$ & $-4.49 \mathrm{E}-11$ \\
$(2.93 \mathrm{E}-11)^{* * *}$ & $(1.61 \mathrm{E}-11)^{* * *}$ \\
MBD & {$[4.56 \mathrm{E}-11]^{* * *}$} & {$[4.56 \mathrm{E}-11]$} \\
& -0.08781 & 0.07948 \\
Gasto en Salud & $(0.70560)$ & $(0.40884)$ \\
& {$[0.0734]$} & {$[0.0734]$} \\
Constante & -0.02998 & -0.05665 \\
& $(0.0315)$ & $(0.0180)^{* * *}$ \\
& {$[0.0335]$} & {$[0.0335]$} \\
4.234058 & 3.407 \\
& $(0.12515)^{* * *}$ & $(0.0808)^{* * *}$ \\
& {$[0.14131]^{* * *}$} & {$[0.141]^{* * *}$} \\
\hline
\end{tabular}

$\mathrm{N}=260$

$*, * *$ y $* * *$ : significativos al 10,5 y $1 \%$ respectivamente.

Desvíos estándar entre paréntesis, robustos entre corchetes.

V.c.2. Mínimos cuadrados en 3 etapas (MC3E):

En la Tabla 5 se pueden observar los resultados de las estimaciones en el marco de MC3E. Tanto el nivel educativo del país, como su migración neta y el gasto público en salud resultan ser variables significativas a la hora de explicar.

A diferencia de lo sucedido en el ámbito de estimaciones por panel, aquí sí resultaron ser significativas las variables que se seleccionaron para explicar MBD. 
Mayor gasto público en salud logra mermar el proceso de MBD, dado que ahora el signo es negativo, contrario a lo que sucedía en estimaciones de panel previas, mientras que a mayor migración y nivel educativo del país el proceso de fuga aumenta.

La presente especificación puede resultar más adecuada (o haber obtenido mejores resultados) que datos de panel, porque es probable que las endogeneidades que existen entre las variables macroeconómicas seleccionadas sea muy alta. De esta manera, un sistema múltiple de ecuaciones estaría captando mejor los efectos.

Como conclusión de las estimaciones, se pueden destacar dos puntos. Por un lado, parece ser más apropiado plantear un sistema como se mencionó anteriormente, quizás pensando en que la especificación puede estar omitiendo variables relevantes. Por otra parte, el trabajo queda supeditado a que tan buena proxy de la fuga de cerebros en general es la fuga de cerebros en el sector salud.

Tabla 5. MC3E

\begin{tabular}{|c|c|c|c|c|}
\hline DV & $\begin{array}{c}\text { Migración } \\
\text { Neta }\end{array}$ & Educación & Crecimiento & MBD \\
\hline
\end{tabular}

$\begin{array}{cc}\text { Diferencial PBI - } & 5.2817 \\ \text { OCDE } & (5.69469) \\ \text { Población } & -0.0024 \\ & (0.0009)^{* *}\end{array}$

Crec. Poblacional $-1058.83$

$(1169.092)$

Gasto Publico (ed. terciaria + salud)

Gasto Publico (ed. terciaria)

Gasto Publico (salud) 
Remesas

Migración Neta
$-0.788$

$$
(0.13)^{* * *}
$$

$\begin{array}{ll}-0.000 & 0.000\end{array}$

3.37E-07

$$
(3.91 \mathrm{E}-0)^{* * *}
$$

$(2.5 \mathrm{E}-06)^{* * *}$

$(1.1 \mathrm{E} 07)^{* * *}$

Educación

Crecimiento

$$
\begin{gathered}
0.9218 \\
(0.162) * * *
\end{gathered}
$$

Constante

61.99

1004.438

$$
(2.1957)^{* * *}
$$

chi2

21.07

15.1

48.12

20.03

0.0005

Errores Estándar entre paréntesis

$*, * *, * * *$ : significatividad al 10,5 y $1 \%$ respectivamente

\section{CONCLUSIONES}

La migración calificada es uno de los temas de mayor interés tanto para los países expulsores de recursos como para países receptores.

Los efectos de la fuga de cerebros son considerados como positivos desde el punto de vista de las remesas, de la transmisión de conocimientos y habilidades y desde la posibilidad de que se visualice el fenómeno brain gain. Por otro lado, la mayor parte de la literatura considera la migración calificada como negativa para los países de origen porque la misma significa una gran pérdida de recursos, dado que gran parte de la inversión que el país realiza en su sistema educativo se evacua con la migración y, por otro lado, se considera negativo el posible desmantelamiento de recursos en áreas centrales para el desarrollo económico de un país, como ingenieros, técnicos, médicos, etc.

Dentro de los determinantes de la fuga de cerebros podemos encontrar la imposibilidad que encuentran los profesionales de ubicarse en áreas bien pagas en el país de origen y las mejores condiciones de contratación y trabajo que se visualizan en países desarrollados, según la revisión bibliográfica realizada. El profesional que tiene dentro de sus posibilidades la migración se compara con co- 
legas dentro del mismo país que están en mejores condiciones que él y con colegas en otros países que tienen condiciones de contratación imposibles de alcanzar en su país de residencia.

En nuestro trabajo se buscó estimar la fuga de cerebros en el sector salud para 20 economías latinoamericanas. El objetivo central del trabajo era buscar los determinantes del fenómeno como así también determinar si la fuga de cerebros en el sector salud tenía un efecto determinante en el desarrollo económico.

Al realizar las estimaciones respecto a la variable Medical Brain Drain (se define como la fuga de cerebros en medicina en el momento i para el país $t$ ), encontramos todas las variables no significativas (Gasto Publico en Salud (\%PBI), Remesas recibidas (\%PBI), Crecimiento del PBI (\%), Número de patentes solicitadas por residentes, Número de artículos publicados en revistas científicas y gasto público en el sector educativo terciario (\%PBI)), y cuando la exigencia en significatividad es más laxa la variable más robusta es remesas.

Por otro lado, se estimaron dos modelos donde las variables dependientes son el logaritmo de la esperanza de vida y el logaritmo de la mortalidad infantil. Estos dos últimos modelos se intentan explicar por el gasto público en salud, el crecimiento, y la variable MBD. En este caso, la variable MBD no resulta estadísticamente significativa, por lo cual se puede inferir que la fuga de cerebros en el sector salud no es un determinante de excepcional importancia para el desarrollo económico de los países latinoamericanos.

Más allá de la especificación seleccionada, la cual puede estar omitiendo variables (como el rol educativo en la mortalidad infantil), la fuga de cerebros de la rama de medicina no logra ser explicada satisfactoriamente por las variables incorporadas ni logra explicar las variables de salud elegidas. Sólo se consiguen significatividades del 5 o 10\% en algunos casos de variables explicativas de MBD, pero se pierden cuando se pasan a estimar los paneles con errores robustos o bootstrap.

Finalmente, debido al resultado que obtuvimos podemos pensar que el MDB puede explicarse mayoritariamente por las condiciones de los países de destino respecto a las que encuentran los profesionales de la salud en sus países natales.

$\mathrm{Al}$ estimar por $\mathrm{MC} 3 \mathrm{E}$, tomando como otras variables dependientes del sistema a la migración neta, la educación y el crecimiento, se obtuvo que el gasto público en salud impacta negativamente en MBD, por lo que se puede pensar en la posible aplicación de un instrumento de política que frene la fuga de cerebros. 
En este caso especifico, se trata del gasto en salud, pero se podría extrapolar este resultado al resto de las ramas, y pensar que mayor apoyo estatal de las distintas ramas del conocimiento, sentarían las bases para que se mantenga el capital humano en su país de formación, evitando así que migre por mejores condiciones.

Debido a los resultados obtenidos en este primer trabajo, queda pendiente para futuras investigaciones incluir en nuestras especificaciones, alguna manera de medir las economías de América Latina en correlato con aquellos países que son receptores de su fuga de cerebros, como así también incluir otras variables explicativas que estén relacionadas con la migración calificada.

\section{REFERENCIAS BIBLIOGRAFICAS}

Bahargava, A., Docquier, F. \& Moullan, Y.(2010). Modeling the effects of physician emigration on human development. Recuperado de http://search.worldbank. org $/$ data?qterm $=$ medical + brain + drain\&_topic_exact $\% 5 \mathrm{~B} \% 5 \mathrm{D}=$ Migration

Beine, M., Docquier, F. \& Rapoport, H. (2001). Brain Drain and Economic Growth: Theory and Evidence, Journal of Development Economics, 64 (1), 275-89

Docquier, F., Lohest, O. \& Marfouk, A. (2005). Brain Drain in Developing Regions (1990-2000), IZA Discussion Papers 1668.

Investigación en Migración Internacional en América Latina (IMILA). Centro de demografía de América Latina. División de Población de la CEPAL (CELADE).

Lim, L. L. (1998). The processes generating the migration of women, Technical Symposium on International Migration and Development, The Hague, Netherlands, 29 June - 3 July, United Nations Administrative Committee of the Coordination Task Force on Basic Services for All, Working Group on International Migration.

Massey, D., Arango, J., Graeme, H., Kouaocuci, A., Pelegrino, A., \& Taylor, J. E. (2000). Teorías sobre la migración internacional: una reseña y una evaluación, Trabajo, 2(3), 5-50.

Mountford, A. (1997). Can a brain drain be good for growth in the source economy, Journal of Development Economics, (53), 287-303

Novick, S. (2008). Migración y políticas en Argentina: tres leyes para un país extenso (1876-2004). Las migraciones en América Latina. Políticas, culturas y estrategias. Recuperado de http://webiigg.sociales.uba.ar/pobmigra/ archivos/Novick8-8-08.pdf

Pellegrino, A. (2000). Drenaje, movilidad, circulación: nuevas modalidades de la migración calificada. Recuperado de http://www.eclac.org/publicaciones/ 
$\mathrm{xml} / 2 / 8852 / \operatorname{lcg} 2124 \mathrm{P} 4$ 4.pdf

Pellegrino, A \& Vigorito, A. (2009). La emigración calificada desde América Latina y las iniciativas nacionales de vinculación. Un análisis del caso uruguayo, Pensamiento Iberoamericano (4), 189-215.

Portes, A. (2007). Migración de desarrollo: una revisión conceptual de la evidencia. En S. Castles, y R. Delgado Wise. Migración y desarrollo: perspectivas desde el sur. México: Universidad Autónoma de Zacatecas.

Samuel, W. (2000). Migration and remittances: a case study of the Caribbean, ponencia presentada al Simposio sobre migración internacional en las Américas, en CEPAL/CELADE/FNUAP/OIM/BID.

Sassen Koob, S. (1988). The mobility of Labor and Capital. Cambridge: Cambridge University Press.

Villa, M., \& Martínez, J. (2001). El mapa migratorio internacional de América Latina y el Caribe: patrones, perfiles, repercusiones e incertidumbres. Centro Latinoamericano y Caribeño de Demografía (CELADE), Santiago, inédito.

(C) 2014 por los autores; licencia otorgada a la Revista Estudios Económicos. Este artículo es de acceso abierto y distribuido bajo los términos y condiciones de la licencia Atribución-No Comercial 3.0 Unported (CC BY-NC 3.0) de Creative Commons. Para ver una copia de esta licencia visite http://creativecommons.org/licenses/by-nc/3.0/ 